\title{
Abuso Físico Infantil: Avaliando Fatores de Risco Psicológicos em Cuidadores Notificados
}

\author{
Child Physical Abuse: Evaluating Psychological Risk Factors \\ in Accused Caregivers
}

\author{
Lilian Paula D. Bérgamo \& Marina Rezende Bazon* \\ Universidade de São Paulo, Ribeirão Preto, Brasil
}

\begin{abstract}
Resumo
Verificou-se em que medida variáveis cognitivas e afetivas/emocionais diferenciariam cuidadores notificados por abusos físicos (G1) de cuidadores sem esse histórico (G2). O Child Abuse Potential Inventory (CAP) foi utilizado para avaliar fatores de risco psicológicos em cuidadores. Um Questionário de Caracterização sócio-demográfica e outro econômico também foram empregados para equiparar os grupos. G1 apresentou um potencial de risco superior a G2, e maiores níveis de Angústia, Rigidez, Problemas com a Criança e Consigo, Problemas com os Outros, e um menor nível de Força do Ego. Essas variáveis se articulam para compor o risco de abuso físico, pois segundo o Modelo do Processamento da Informação Social, remeteriam a processos básicos cognitivos/afetivos subjacentes a percepções e avaliações/interpretações, associados ao comportamento parental abusivo.

Palavras-chave: Maus-tratos, abuso físico, percepção, avaliação/interpretação, modelo do processamento da informação social.
\end{abstract}

\begin{abstract}
It was verified to what extent cognitive and affective/emotional variables could distinguish caregivers accused of committing physical abuse (G1) from those without physical abuse records (G2). The Child Abuse Potential Inventory (CAP), which is an instrument designed to assess psychological risk factors in caregivers, was used. A questionnaire on socio-demographic characterization and another on economic classification were also employed to equate the groups. G1 presented a greater potential risk than G2, higher levels of Distress, Rigidity, Problems with the Child and with Themselves, Problems with Others, and a lower level of Ego Strength. These variables contribute with the composition of physical abuse risk, since, in agreement with the Social Information Processing Model, they would be related to cognitive and affective basic processes which are veiled to the perceptions and evaluation/interpretations, associated to abusive parental behavior.

Keywords: Maltreatment, physical abuse, perception, evaluation/interpretation, social information processing model.
\end{abstract}

A violência intrafamiliar, especialmente a praticada contra crianças, vem se tornando alvo de atenção na comunidade científica, tanto pela grande prevalência do fenômeno quanto pela gravidade de suas conseqüências. A World Health Organization (WHO) e a International Society for Prevention of Child Abuse and Neglect (ISPCAN, 2006) denominam esta forma de violência como "maus-tratos infantis" e indicam a existência de quatro principais tipos ou modalidades: abuso físico, abuso psicológico, negligência e abuso sexual, sendo foco deste estudo somente o abuso físico. De acordo com es-

\footnotetext{
* Endereço para correspondência: Departamento de Psicologia e Educação, Faculdade de Filosofia, Ciências e Letras, Universidade de São Paulo, Av. Bandeirantes, 3900, Monte Alegre, Ribeirão Preto, SP, Brasil 14040901. Tel: (16) 3602-3830 ou 3602-3739. E-mail: mbazon@ffclrp.usp.br
}

ses organismos, esse tipo pode ser definido como o "uso intencional da força física contra a criança que resulta, ou tem alta possibilidade de resultar em dano a sua saúde, sobrevivência, desenvolvimento e dignidade. Os atos agressivos incluem pancadas, surras, mordidas, estrangulamentos, queimaduras, envenenamentos e sufocamentos" (p. 10).

Segundo Belsky (1993), a manifestação dos maus-tratos em cada uma de suas modalidades é um fenômeno complexo, pois envolve em sua etiologia variáveis diversas, pertencentes a diferentes sistemas, que se influenciam mutuamente.

No nível do microssistema haveria variáveis ligadas às características psicológicas e comportamentais dos membros da família que, em conjunto com outras, atuariam para compor o risco e a emergência da problemática. Dentro disso, em meio a uma gama de variáveis, as in- 
vestigações destacam, para o abuso físico, as seguintes variáveis: percepções e atribuições negativas em relação ao comportamento da criança, expectativas irrealistas quanto às capacidades/comportamento da criança, pouca flexibilidade em entender o seu comportamento e pouca capacidade de selecionar estratégias adequadas de manejo, baixa auto-estima e força do ego, altos níveis de angústia e depressão, infelicidade, queixas somáticas, sentimentos de incompetência, tendência à desordem/raiva, impulsividade, implementação de estratégias duras de disciplina (Black, Heyman, \& Slep, 2001; Milner \& Chilamkurti, 1991).

Investigações nacionais, seja com amostras populacionais ou com grupos clínicos, também destacam que determinados aspectos psicológicos dos cuidadores se constituem em risco para abuso físico no contexto sociocultural brasileiro. Ribas, Ribas e Valente (2006), avaliando indicadores de bem-estar emocional numa amostra de pais da população geral, verificaram que sintomas depressivos estavam associados a uma auto-percepção menos positiva concernente ao papel parental (menor satisfação com o seu exercício e menos competência na sua realização), e a interações parento-filiais mais ásperas, as quais envolviam para $13 \%$ dos entrevistados, a implementação de práticas de punição física. Vitolo, Fleitlich-Bilyk, Goodman e Bordin (2005) encontraram que as famílias que empregavam punição física grave possuíam um histórico de problemas de saúde mental, estando esses associados a problemas de saúde mental apresentados pelas crianças. De Antoni, Barone e Koller (2007) avaliando indicadores de risco e de proteção em famílias fisicamente abusivas sublinharam que para algumas delas havia relatos de sintomas depressivos e de descontrole emocional, sendo que alguns dos cuidadores haviam, efetivamente, sido diagnosticados como portadores de desordem psiquiátrica.

Com base na identificação dos fatores associados ao abuso físico, concernente às características psicológicas e às relações proximais dos cuidadores, modelos teóricos foram desenvolvidos no intuito de avançar nas explicações sobre o fenômeno. Inspirado na "Teoria do Processamento da Informação Social" (PIS), Joel Milner elaborou uma proposição que descreve as atividades cognitivas que podem subjazer o comportamento de abuso físico infantil, e como estas podem ser impactadas por características de personalidade do cuidador (como depressão e ansiedade), bem como por outros eventos, relativos aos demais níveis ecológicos (Milner, 2003).

O Modelo proposto por Milner envolve quatro estágios, três cognitivos (1- percepções, 2- avaliações e interpretações do comportamento da criança, 3- integração da informação e seleção da resposta) e um cognitivo-comportamental (implementação da resposta e seu monitoramento). A esses se somam esquemas cognitivos (estruturas de informação pré-existentes) que influenciam a forma pela qual o cuidador percebe, interpreta e avalia o comportamento infantil. Acredita-se que pais em alto risco para o cometimento de abuso possuam mais chances de ter déficits em seus esquemas cognitivos e crenças rígidas relativas ao comportamento da criança e suas habilidades, assim como em relação às práticas de criação e às próprias habilidades parentais (Milner, 2000, 2003).

No presente trabalho foram focalizados especificamente os processos básicos, segundo o Modelo acima descrito. De acordo com Milner (2003), cuidadores com alto risco para o abuso físico teriam percepções relativas ao comportamento infantil apresentando mais distorções ou erros, quando comparados a cuidadores não abusivos. Outras investigações apontam que o grupo de alto risco tende a perceber mais desobediência por parte da criança, assim como mais problemas de comportamento nessas (Dopke, Lundahl, Dunsterville, \& Lovejoy, 2003; Milner, 1994). No entanto, Haskett, Scott, Grant, Ward e Robinson (2003) alertam sobre a necessidade de se investigar a influência dos comportamentos problemáticos que as crianças efetivamente possam apresentar e a das características parentais (tais como angústia e depressão) na composição do risco de abuso físico.

No que diz respeito às interpretações/avaliações do comportamento da criança, pais abusivos realizariam julgamentos quantitativa e qualitativamente diferentes de outros pais, tendendo a interpretá-lo como tendo uma intenção hostil e como mais sério e mais danoso (Ateah \& Durrant, 2005; De Paúl, Asla, Pérez-Albéniz, \& De Cádiz, 2006; Milner, 2003). Neste sentido, Montes, De Paúl e Milner (2001) verificaram uma interação entre o status de risco e o tipo de transgressão cometida pela criança, sendo que as transgressões convencionais ou mais comuns para a faixa etária, como, por exemplo, aquelas em que a criança recusa-se a atender prontamente uma solicitação do adulto, como ir tomar banho ou ajudar em determinadas tarefas domésticas, foram avaliadas como mais graves por adultos que compuseram o grupo de alto risco. Além disso, sabe-se que grupos de alto risco sentem mais aversão em relação às transgressões convencionais, o que seria explicado pelo nível de estimulação fisiológica vivenciado por esses cuidadores diante de um estímulo estressante relacionado ao comportamento infantil, como choros, birras e oposição/recusas (De Paúl et al., 2006; Milner, 2003).

Ainda, os pais em risco tenderiam a atribuir os comportamentos negativos da criança ou sua desobediência a fatores internos, estáveis e globais, ou seja, a características inerentes à criança, a qual, em suas percepções, se comportaria daquela forma sempre e em todas as situações. Por essa razão, não se sentiriam como tendo o controle ou capacidade de manejar o comportamento infantil (indicando uma orientação externa de controle), o que estaria relacionado à utilização de estratégias disciplinares coercitivas e ao potencial de abuso (De Paúl et al., 2006; Dopke \& Milner, 2000; McElroy \& Rodriguez, 2008; Montes et al., 2001; Rodriguez, 2010). 
É importante notar que os processos cognitivos que compõem os estágios, de acordo com o Modelo do PIS podem ser influenciados pelas características psicológicas do cuidador, como altos níveis de afeto negativo (raiva, hostilidade) e angústia pessoal (depressão, ansiedade, baixa auto-estima) (Milner, 2000, 2003).

Nesta direção, pais que considerariam o comportamento da criança como sério e intencional ou que o julgassem de forma implacável em razão de o mesmo não expressar a submissão da criança, poderiam experimentar sentimentos de raiva, fazendo aumentar o risco de reagirem por meio da punição física (Ateah \& Durrant, 2005; Dopke et al., 2003). Assim, pode-se afirmar que a raiva e a hostilidade têm sido consideradas como preditores tanto de estilos disciplinares autoritários como do potencial de abuso físico, sobretudo se há déficits no manejo adequado dessas emoções (Hien, Cohen, Caldeira, Flom, \& Wasserman, 2010) e se uma orientação externa de controle prepondera, havendo a percepção de que a criança é quem exerce controle nas interações pais-criança (Francis \& Wolfe, 2008; Milner, 2000; Rodriguez, 2010; Rodriguez \& Richardson, 2007).

Em se tratando da angústia pessoal vivida pelo cuidador em seu contexto de vida e nas interações com a criança, novamente se observam inter-relações significantes entre variáveis de natureza afetiva e cognitiva para a produção dos abusos, já que altos níveis de angústia parental têm sido associados com padrões negativos de atitudes e crenças relacionadas à criança (Haskett et al., 2003). Pittman e Buckley (2006) encontraram que a angústia seria um fator mais significativo para as mulheres que para os homens, os quais, em seu turno, apresentariam maior rigidez em relação ao comportamento da criança e às práticas parentais a serem adotadas.

A angústia também tem sido atrelada a baixos níveis de empatia, normalmente apresentados por cuidadores em alto risco de abuso. Hipotetiza-se que o estado de angústia seria um dos fatores responsáveis pela dificuldade em se colocar na perspectiva do outro, principalmente em situações em que estaria em questão a expressão de sentimentos de sofrimento. Segundo o modelo do PIS, tal dificuldade influenciaria a avaliação e a interpretação do comportamento do outro (De Paúl, Pérez-Albéniz, Guibert, Asla, \& Ormaechea, 2008; Francis \& Wolfe, 2008).

Para Milner (1986), a depressão parental é um dos componentes desse estado de angústia e tem sido associada a práticas de punição física e ao potencial de abuso (Mammen, Kolko, \& Pilkonis, 2002; Milner \& Chilamkurti, 1991; Palusci, Smith, \& Paneth, 2005; Woodward \& Fergusson, 2002). Todavia, segundo Shay e Knutson (2008), o efeito exercido pela depressão na produção do abuso físico parece ser indireto, ou seja, a relação entre tais variáveis seria mediada por sentimentos de raiva/ irritabilidade. De todo modo, a depressão parental geralmente está associada a ações disciplinares que tendem a se agravar, o que aumenta as chances de comportamentos inadequados nas crianças, os quais, por sua vez, incre- mentam sentimentos de raiva/irritabilidade e o afeto deprimido nos pais.

Ainda no tocante às variáveis de natureza afetiva, sabese que cuidadores considerados em alto risco para o abuso físico tenderiam a se sentir instáveis emocionalmente e pouco adequados nas suas relações interpessoais, o que indicaria baixo nível de força egóica e, dentro disso, de auto-estima (Milner, 1986). Estudos demonstraram que estes fatores podem influenciar negativamente as percepções em relação à criança, dificultando, inclusive, o reconhecimento de suas emoções, e a maneira de lidar com as situações de estresse (Balge \& Milner, 2000; Milner, 1994). Nessa mesma linha, a baixa tolerância à frustração também tem sido considerada um fator associado ao risco de abuso físico por interferir negativamente na percepção dos pais a respeito dos comportamentos da criança e das suas interações com ela (McElroy \& Rodriguez, 2008).

Por fim, vale destacar o papel de duas variáveis contextuais que impactariam os processos cognitivos básicos subjacentes ao comportamento dos pais por abusarem fisicamente das crianças o estresse parental e o apoio social. O estresse parental vivido na interação com a criança é um fator fortemente associado tanto a estilos disciplinares autoritários como ao potencial de abuso (Francis \& Wolfe, 2008; Rodriguez, 2010; Rodriguez \& Richardson, 2007) por influenciar os processos cognitivos dos cuidadores (Milner, 2003). Aponta-se que a fonte principal de estresse para pais notificados por abuso físico é a percepção negativa dos comportamentos da criança e de suas características, e que os comportamentos de desobediência por parte desta são considerados como altamente estressantes porque são percebidos como ameaçadores e incontroláveis (DiLauro, 2004; Dopke \& Milner, 2000).

$\mathrm{O}$ apoio social percebido/recebido, em seu turno, pode atuar como uma variável que modera as adversidades vividas pelos cuidadores, principalmente nessas situações consideradas estressantes (Jackson, 2000; Suzuki, 2010). De outra forma, a existência de problemas nas relações sociais e, por conseqüência, o isolamento social pode aumentar o risco de práticas abusivas contra os filhos, pois, além da falta de ajuda, em termos práticos, padecerse-ia da falta de parâmetros em termos de exemplos de como lidar com as situações de conflito e, mais especificamente, da possibilidade de problematizar e compartilhar com outros cuidadores, no entorno comunitário, as angústias relativas às exigências que o papel parental impõe (Ochotorena \& Madariaga, 1996; Pittman \& Buckley, 2006). Assim, cuidadores que cometem abuso físico geralmente relatam baixo nível de apoio emocional, no sentido de contar com pouca compreensão e empatia por parte de outros, e de apoio instrumental no sentido de não serem ajudados no cuidado com a criança (Coohey, 1996).

Considerando o panorama acima descrito e tendo por base o Modelo PIS proposto por Milner, o presente tra- 
balho teve por objetivo verificar em que medida aspectos cognitivos, afetivos/emocionais e contextuais diferenciariam, na realidade sociocultural brasileira, cuidadores notificados por abusos físicos de cuidadores sem histórico dessa problemática, aferindo assim, características específicas do grupo clínico quanto aos processos básicos "percepção e avaliação/interpretação" associados à produção do problema em foco, o abuso físico infantil.

\section{Método}

\section{Participantes}

Participaram do estudo 60 cuidadores, 30 constituíram o grupo clínico (G1) referente a cuidadores notificados ao Conselho Tutelar por abusos físicos e 30 constituíram o grupo de comparação (G2) referente a cuidadores sem histórico da problemática. Os participantes do G1 foram selecionados a partir das notificações realizadas aos Conselhos Tutelares em uma cidade do interior do Estado de São Paulo, retendo-se aqueles casos que correspondiam à definição de abuso físico adotada nesta investigação, relativos a crianças/adolescentes com até 15 anos de idade, visto que esta modalidade de maus-tratos acontece raramente com adolescentes mais velhos.

Do total dos casos selecionados (90), houve perdas significativas por razão de mudanças de endereço e/ou endereços não localizados (44 casos) e algumas recusas (8). Outros 8 casos não foram contatados em função de a amostra ter atingido o número pretendido para a composição de $\mathrm{G} 1(n=30)$.

Os participantes do G2 foram selecionados a partir do contato e do levantamento de informações junto a famílias conhecidas e indicadas por psicólogos e assistentes sociais atuando em programas da assistência social, nas mesmas comunidades em que residiam os participantes do G1, tendo por base o critério de os cuidadores serem considerados "adequados" e terem bom relacionamento com os filhos. Para a composição do grupo, procedia-se à aplicação de um questionário de caracterização com vistas ao pareamento de G2 com G1 em variáveis sóciodemográficas como idade, sexo, escolaridade, nível sócio-econômico, situação conjugal, total de crianças sob os cuidados e idade de um dos filhos (a criança em função da qual a notificação teria sido feita). Ademais, baseando-se nos apontamentos de Gershoff (2002, citado por Gomide, 2006), verificava-se aspectos das práticas parentais, no sentido de excluir abusos físicos, pesquisando a presença/ausência dos seguintes indicadores: punir fisicamente a criança, numa freqüência superior a duas vezes ao mês, usar objetos para punir (cintas, chinelos, etc.) e produzir ferimentos. Com isso, 40 cuidadores foram contatados, sendo que 4 recusaram colaborar com o estudo e 6, embora tendo colaborado, após uma análise preliminar dos dados coletados, não atendiam aos critérios estabelecidos, especificamente em relação às variáveis de pareamento, tendo seus dados sido desprezados para o estudo.
Considerando um $p<0,05$, os participantes do presente estudo caracterizaram-se por terem em média 34 anos em G1 e 36 em G2 $(p=0,373) ; 20 \%(n=6)$ serem do sexo masculino e $80 \%(n=24)$ do feminino, em ambos os grupos; $70 \%(n=21)$ possuírem companheiro(a) também em ambos os grupos; terem escolaridade média de 6,2 anos em G1 e 6,5 anos em G2 $(p=0,765)$. Os participantes de G1 tinham em média 2,9 filhos com média de idade de 9,7 anos, e em $\mathrm{G} 2$ o número médio de filhos era de 2,5 com média de idade de 9,2 anos ( $p=0,363$ e $p=0,603$ respectivamente). Em ambos os grupos, os participantes, eram majoritariamente de grupos familiares pertencentes à classe $\mathrm{C}(\mathrm{G} 1=57 \% \mathrm{e} \mathrm{G} 2=63 \%, p=0,117)$, na qual a renda familiar estimada gira em torno de dois salários mínimos.

\section{Instrumentos}

O principal instrumento utilizado foi o Child Abuse Potential Inventory (CAP; Milner, 1986). De origem norte-americana, foi construído com base em uma ampla revisão de literatura teórica e empírica sobre fatores correlacionados ao abuso físico e à negligência, enfatizando-se traços de personalidade e problemas interpessoais (Milner, 1986). O CAP tem se mostrado útil na discriminação de grupos de abuso físico; abuso físico e negligência; alto e baixo risco para os maus-tratos, sendo especialmente eficaz para identificar casos de abuso físico moderados e graves (Bringiotti, 1999; Milner, 1986).

Quanto às suas qualidades psicométricas, Milner (1994) obteve estimativas de teste-reteste para a escala de Abuso com intervalos de um dia, uma semana, um mês e três meses, sendo que os índices de fidedignidade encontrados foram de $0,91,0,90,0,83,0,75$, respectivamente. Pela estimativa da consistência interna do CAP (método Kuder-Richardson-20) o autor encontrou índices de 0,92 para grupos de comparação, 0,91 a 0,94 para grupos de cuidadores negligentes, e 0,95 a 0,96 para grupos de abusadores físicos (Milner, 1986). Por conta dessas qualidades, o CAP já foi traduzido e adaptado em vários países (Bérgamo, Pasian, Ávila de Mello, \& Bazon, 2009).

No Brasil, estudos também têm atestado suas qualidades psicométricas. Bérgamo et al. (2009) avaliaram sua fidedignidade (consistência interna) chegando a estimativas adequadas para a escala de Abuso ( 0,92 para grupos clínico e de comparação, respectivamente), para as dimensões de Angústia (0,94 e 0,93), Rigidez (0,75 e 0,83), Força do Ego (0,93 tanto para grupo clinico como de comparação) e Solidão $(0,81$ e 0,80$)$. Observou-se também que 56 itens do CAP discriminaram dois grupos contrastantes quanto ao risco de abuso infantil, sendo que desses 56 itens, 35 pertenciam à escala de Abuso. Rios (2010), com vistas à adaptação transcultural do CAP para o Brasil, verificou que a versão em português apresenta uma equivalência semântica adequada e demonstrou a validade de constructo do instrumento por meio da Análise 
Fatorial de Componentes Principais. A autora também avaliou a fidedignidade do CAP por meio de sua consistência interna (alfa de Crombach), chegando ao índice de 0,95 .

No presente estudo utilizou-se a versão preliminar em português produzida no Brasil por Ávila de Mello (2008, citado por Rios, 2010), a qual foi aplicada no formato de entrevista dado o baixo nível de escolaridade de grande parte dos participantes, cuidando-se para não fornecer explicações ou fazer comentários durante a aplicação (Milner, 1986). O inventário é constituído por 160 itens aos quais a pessoa responde se concorda ou discorda da afirmação. A Escala de Abuso, principal do CAP, possui 77 itens os quais também compõem as seis dimensões do instrumento. A dimensão Angústia (36 itens) refere-se à presença de problemas de ajustamento pessoal no cuidador, correspondente a sentimentos de frustração, tristeza, solidão, depressão, preocupação, confusão, rejeição, baixa auto-estima e raiva. A dimensão Rigidez (14 itens) identifica um estilo parental rígido, embasado por crenças e expectativas rígidas quanto ao comportamento da criança. A dimensão Infelicidade (11 itens) refere-se à existência de uma polaridade de conteúdo felicidade/infelicidade frente à vida em geral e a determinados aspectos. A dimensão Problemas com a Criança e Consigo (6 itens) refere-se à tendência a perceber a criança de maneira negativa, com habilidades limitadas e com poucas competências, além de avaliar a percepção do cuidador à respeito de suas próprias habilidades. A dimensão Problemas com a família (4 itens) avalia dificuldades relativas às relações estabelecidas com a família extensa. A dimensão Problemas com os outros (6 itens) também avalia se os cuidadores consideram as relações sociais difíceis. Há ainda as escalas que detectam distorções nas respostas: Inconsistência Interna, Resposta ao Acaso, Desejabilidade Social ou Mentira. Os 160 itens do CAP foram posteriormente agrupados por Milner (1986) em duas escalas especiais, as quais estão em processo de validação e normatização. Uma delas refere-se à avaliação da "Solidão" (15 itens), averiguando o conhecimento dos respondentes com relação às suas possibilidades de ajuda; e a outra, denominada de "Força do Ego" (40 itens), busca avaliar a percepção do adulto sobre sua estabilidade emocional e sentimentos de adequação nas relações interpessoais. Vale dizer que alguns itens do CAP compõem mais de uma escala e que tanto o posicionamento de acordo como de desacordo são pontuados com pesos diversos, os quais fazem parte de um "crivo avaliativo" específico (J. S. Milner, comunicação pessoal, 03 de maio de 2007).

Segundo Milner (1986, 1990), os resultados devem ser interpretados em termos gerais, sendo que quanto mais alto o valor na escala de Abuso maior o potencial do cuidador em termos de risco de maus-tratos. Escores totais, nessa escala, igual ou superior a 215 pontos (dos 486 possíveis, relativos ao conjunto de 77 itens desta escala) identificariam cuidadores apresentando risco de abuso infantil.

Dois outros instrumentos foram utilizados tendo em vista o pareamento entre os grupos de participantes. Um deles é o "Questionário de Caracterização sócio-demográfica", que foi elaborado com base no modelo proposto por Bringiotti (1999), visando obter informações em termos de idade, sexo, nível educacional, situação conjugal, número de filhos ou crianças sob os cuidados, e idade dessas. O outro é o "Critério de Classificação Econômica Brasil - CCEB", desenvolvido a partir de um estudo realizado com base no banco de dados do "Levantamento Sócio-Econômico" (LSE), de 1993, do IBOPE (Associação Brasileira de Empresas de Pesquisa [ABEP], 2003). Esse caracteriza-se como um check-list pelo qual verifica-se a posse de determinados objetos ou bens de consumo e o grau de instrução do chefe da família, de modo a poder classificar a família, em termos econômicos, em classes que vão de $\mathrm{A} 1 \mathrm{a} \mathrm{E}$, conforme o total de pontos alcançados (de 0 a 34 ).

\section{Procedimentos de Coleta e de Análise dos Dados}

Antes de iniciar a investigação, o projeto de pesquisa foi submetido e avaliado pelo Comitê de Ética em Pesquisa com Seres Humanos, recebendo parecer favorável (Processo CEP - Faculdade de Filosofia, Ciências e Letras de Ribeirão Preto [FFCLRP] n ${ }^{\circ}$ 257/20062006.1.827.59.0). Na sequência, os potenciais participantes passaram a ser contatados em suas residências, solicitando-lhes a colaboração com o estudo, e apresentando-lhes o Termo de Consentimento Livre e Esclarecido, no caso de aceite. Aos participantes do G1 apresentou-se também uma carta que continha a autorização dos Conselhos para contatá-los e que ressaltava a desvinculação da pesquisa das práticas daquele órgão. A aplicação dos instrumentos durou cerca de 50 minutos com cada participante.

As respostas fornecidas ao Questionário de Caracterização foram analisadas e convertidas em categorias numéricas, bem como a avaliação feita com a aplicação do CCEB. As respostas fornecidas ao CAP foram corrigidas segundo a pontuação de cada item fornecida pelo autor e compiladas em banco de dados, procedendo-se, inicialmente, ao cálculo de médias e desvios padrão. Em seguida, os resultados foram analisados estatisticamente utilizando-se o teste t de Student para amostras independentes (quando a distribuição da variável era considerada normal) ou Mann-Whitney Rank Sum test (quando a distribuição não era normal). Diferenças significativas foram consideradas adotando-se um $p<0,05$.

\section{Resultados}

A aplicação do CAP forneceu dados cuja análise indicou diferenças significativas entre os grupos na maioria das dimensões que compõem a escala de Abuso. A tabela a seguir sintetiza tais resultados. 
Bérgamo, L. P. D. \& Bazon, M. R. (2012). Abuso Físico Infantil: Avaliando Fatores de Risco Psicológicos em Cuidadores Notificados.

Tabela 1

Comparação dos Grupos em Relação às Escalas do CAP

\begin{tabular}{lrrrrr}
\hline \multirow{2}{*}{ Escalas } & \multicolumn{3}{c}{ Grupo clínico - G1 } & \multicolumn{3}{c}{ Grupo de comparação - G2 } \\
& \multicolumn{2}{c}{$n=30$} & \multicolumn{2}{c}{$n=30$} \\
& Média & $D P$ & Média & $D P$ & $t / T$ \\
\hline Abuso & 223,87 & 99,3 & 166,90 & 83,34 & $2,40^{*}$ \\
$\quad$ Angústia & 133,40 & 76,16 & 95,03 & 68,58 & $2,05^{*}$ \\
$\quad$ Rigidez & 42,53 & 16,37 & 30,07 & 20,40 & $1080,50^{* *}$ \\
$\quad$ Infelicidade & 15,77 & 11,91 & 16,97 & 12,71 & \\
$\quad$ Problemas com a criança e consigo & 4,83 & 5,31 & 2,47 & 3,59 & $1048,50^{*}$ \\
$\quad$ Problemas com a família & 12,63 & 11,57 & 9,00 & 9,73 & $2,21^{*}$ \\
$\quad$ Problemas com os outros & 17,10 & 6,48 & 13,53 & 5,96 & $-2,21^{*}$ \\
Força do ego & 17,47 & 9,75 & 23,07 & 9,88 & \\
Solidão & 8,80 & 3,77 & 7,17 & 3,62 & 3,39 \\
Desejabilidade social ou mentira & 9,57 & 3,44 & 9,67 & 1,43 & \\
Acaso & 3,30 & 1,66 & 3,77 & 1,10 & \\
Inconsistência interna & 1,50 & 1,31 & 1,37 & & \\
\hline
\end{tabular}

${ }^{*} p<0,05 ; * * p<0,01$.

Pelo exposto na Tabela 1, verifica-se que a escala de Abuso e as dimensões Angústia, Rigidez, Problemas com a criança e consigo e Problemas com os outros indicaram diferenças entre os grupos, sendo que G1 obteve médias superiores a G2, o que revela a presença de maiores dificuldades nestas áreas para os participantes do primeiro grupo.

Quanto à escala Força do ego, a média de G1 foi menor do que a de G2, o que era esperado, pois o construto avaliado por esta escala se constitui em um fator de proteção e não em um de risco, indicando que os participantes de G1 possuem maior fragilidade nesse aspecto se comparado a $\mathrm{G} 2$.

\section{Discussão}

Nos resultados obtidos, destaca-se que os escores médios obtidos na escala de Abuso do CAP apontou maior potencial de risco em G1 que em G2, o que permite dizer que os cuidadores notificados por abusos físicos apresentariam com mais intensidade traços e estilos parentais característicos de cuidadores que maltratam fisicamente os filhos (Milner, 1990). A maioria das dimensões que compõe tal escala diferenciou os grupos estudados, indicando que as variáveis representam aspectos específicos do grupo de cuidadores notificados.

Esmiuçando os resultados, verifica-se que os grupos se diferenciaram nos níveis de rigidez, sendo essa dimensão a que mais contribuiu para compor o potencial de risco em G1. Seguindo a lógica do Modelo do Processamento da Informação Social proposto por Milner (2000, 2003), a rigidez estaria na base das distorções cognitivas que, por sua vez, levariam a falhas no processamento da informação relativa à criança, e ao abuso físico. Assim, os cuidadores de G1 teriam atitudes mais rígidas quanto à aparência e ao comportamento infantil, sendo que na ótica destes, a criança deveria apresentar-se de modo "sempre asseado" e "ser sempre bem comportada e quieta". Neste tocante, supõe-se que a inflexibilidade nas atitudes parentais, pela dificuldade em tomar a perspectiva da criança, concorreria para uma percepção preponderantemente negativa dessa (Najmi, Bureau, Chen, \& Lyons-Ruth, 2009) e esse tipo de percepção seria característico de cuidadores que cometem abuso físico (Dopke et al., 2003; Haskett et al., 2003; Milner, 1994).

Vale ressaltar que a rigidez pode comprometer não só a percepção do comportamento infantil, como também as interpretações, avaliações e atribuições que adultos com alto potencial de risco realizam (Ateah \& Durrant, 2005; De Paúl et al., 2006; Dopke \& Milner, 2000; McElroy \& Rodriguez, 2008; Montes et al., 2001).

É interessante notar que é nesse contexto que os resultados na dimensão Problemas com a Criança e Consigo ganham sentido. Os participantes de G1 pontuaram mais alto que os de $\mathrm{G} 2$, indicando que teriam mais possibilidade de perceber a criança como tendo pouca habilidade e competência ou com algum tipo de problema - lenta, tendo problemas especiais e/ou "se metendo muito em encrencas" (Milner, 1986, 1990). Esses resultados podem ser explicados não só com base nas distorções cognitivas que caracterizam cuidadores abusivos, como também no tipo de estresse que vivenciam, sendo esse ligado prioritariamente às características/comportamentos infantis, visto que tais cuidadores tenderiam a avaliar a criança como sendo de difícil manejo, necessitando de supervisão excessiva, o que aumentaria as chances da implementação de práticas parentais coercivas e abusivas (DiLauro, 2004; Francis \& Wolfe, 2008; Rodriguez, 2010; Rodriguez \& Richardson, 2007). 
Nesse panorama de dificuldades relativas à rigidez das atitudes parentais, que influenciam negativamente o processamento da informação relativa à criança, e de conseqüente aumento de estresse na interação com esta, a percepção de ter apoio social poderia atenuar/moderar essas dificuldades (Jackson, 2000; Suzuki, 2010). No caso dos cuidadores avaliados, não haveria diferença significativa entre os grupos no que respeita às relações com a família estendida, o que equivale dizer que em ambos a percepção do apoio seria equiparável. No entanto, os resultados obtidos na dimensão Problemas com os Outros apontou um nível maior de dificuldades nas relações sociais em G1, indicando que esse perceberia o contato social como causador de mais dificuldade e sofrimento (Milner, 1986). Estudos demonstram que pais com histórico de abuso físico referem que as dificuldades vivenciadas estariam num plano mais subjetivo que objetivo, onde a falta de apoio seria de natureza fundamentalmente emocional, como a necessidade de ser ouvido, aconselhado, compreendido (Coohey, 1996; Heyman \& Slep, 2000).

Deve-se levar em conta, neste contexto, os resultados obtidos nas dimensões Angústia e Força do Ego. Em relação à angústia, G1 a experienciaria num nível mais elevado que G2, apresentando assim um estado emocional mais fragilizado, com sentimentos de depressão, frustração, tristeza, preocupação, confusão, desordem, baixa auto-estima e raiva (Milner, 1986). Investigações têm demonstrado que esses fatores se constituem em risco para o abuso físico (De Antoni et al., 2007; McElroy \& Rodriguez, 2008; Palusci et al., 2005; Pittman \& Buckley, 2006; Vitolo et al., 2005; Woodward \& Fergusson, 2002) quando na presença de fatores de natureza cognitiva (Francis \& Wolfe, 2008; Rodriguez \& Richardson, 2007), e que déficits no controle das emoções é um problema vivido por cuidadores com histórico de abuso (Hien et al., 2010; Mammen et al., 2002).

Ainda, no que se refere à Força do Ego, níveis menos elevados foram identificados em G1, indicando que seus integrantes teriam mais sensações de inadequação nas relações interpessoais do que G2 (Milner, 1990). Dessa forma, hipo-tetiza-se que a angústia e os menores níveis de Força do Ego concorreriam para a percepção das relações sociais como um problema em si e para a produção da própria dificuldade em obter apoio, gerando uma dinâmica de isolamento sócio-emocional e de incremento do estresse experimentado, fatores esses, considerados proximais à emergência do abuso físico (Black et al., 2001).

Tendo como base o Modelo do PIS (Milner, 2000, 2003), cogita-se que a avalanche de sentimentos negativos tornaria o cuidador mais propenso a não reconhecer os sinais emocionais da criança ou a distorcer os comportamentos infantis, no sentido de percebê-los como graves (Balge \& Milner, 2000). Segundo Francis e Wolfe (2008), a dificuldade para interpretar os sinais emocionais da criança, aliada à tendência a percebê-los como hostis, colocaria os cuidadores em alto risco, numa posição de reatividade aos conflitos, o que pode gerar um sentimento de frustração no exercício do papel parental, aumentando as chances de utilizarem a punição.

Além disso, conforme detectado em G1 um estado emocional mais fragilizado, pode-se pensar que esse pode interferir na percepção das próprias capacidades físicas/ saúde para dar conta das demandas da parentalidade. Neste sentido, retomando os resultados obtidos na dimensão Problemas com a Criança e Consigo, tem-se que G1 se sentiria também com mais limitações em relação à própria saúde física que G2. Nessa direção, sabe-se que doenças graves nos cuidadores, bem como a própria percepção de incapacidade física seriam um dos fatores de risco para o abuso de crianças (Brown, Cohen, Johnson, \& Salziger, 1998; Milner, 1994).

Por fim, é necessário tecer algumas considerações a respeito das dimensões que não apresentaram diferenças significativas entre os grupos. $O$ fato de a dimensão Infelicidade não ter diferenciado os grupos remete a hipóteses da ordem do próprio construto. Pensa-se que para o contexto sociocultural brasileiro esta dimensão talvez não represente sentimentos especificamente relacionados aos problemas característicos de famílias envolvidas em situações de maus-tratos, mas represente sentimentos que permeariam o cotidiano de famílias sem grandes dificuldades nas interações com os filhos. É preciso considerar os resultados relativos à consistência interna dos itens deste construto numa amostra brasileira, os quais revelaram índices de fidedignidade abaixo do que seria adequado (Bérgamo et al., 2009).

Já a explicação para a dimensão Solidão não ter indicado diferença entre os grupos parece residir na própria dinâmica dos cuidadores que cometem abuso físico. É possível que os participantes de G1 realmente não desconheçam suas possibilidades de ajuda, principalmente quando se trata de pessoas mais próximas, na família estendida. As dificuldades efetivamente vividas nas interações sociais parecem decorrer de deficiências no provimento de respostas a necessidades de natureza mais subjetiva, como a de serem compreendidos e ajudados nos cuidados com a criança, seja porque tal apoio de fato não existe, seja porque os indivíduos assim percebem a si e ao outro (Coohey, 1996).

Quanto à dimensão Problemas com a Família, o resultado permite dizer que os dois grupos perceberiam dificuldades na mesma proporção. Assim, duas hipóteses são possíveis: a primeira seria relacionada a diferenças na representação desse construto para o nosso contexto, onde os problemas, devido a características socioculturais, só seriam considerados como tais se os conflitos se expressassem de maneira muito contundente, como por exemplo, quando gerassem rompimentos familiares. A segunda hipótese estaria relacionada à desejabilidade social, onde G1 teria fornecido respostas tentando passar 
uma boa imagem, já que os itens que compõem esta dimensão trazem afirmações bem diretas sobre discórdias e conflitos familiares, o que pode ser mais difícil de ser revelado a um entrevistador pouco conhecido.

Ademais, os grupos não se diferenciaram nas escalas de validade, o que era desejado, pois a diferença colocaria em dúvida a confiabilidade das respostas dadas por um deles e, assim, todas as análises comparativas. É interessante notar que, adotando-se as normas americanas, de forma exploratória, as médias obtidas foram baixas, indicando haver pouca probabilidade de as respostas fornecidas pelos participantes apresentarem distorções. Assim, os escores médios obtidos pelos dois grupos ficaram abaixo da nota de corte americana (6) para as escalas de Resposta ao Acaso e Inconsistência Interna, e foram ligeiramente superiores para escala de Desejabilidade Social, para a qual a nota de corte é de 7 (Milner, 1986). Quanto aos escores mais elevados nesta última escala, a hipótese é de que o modo adotado para a aplicação do instrumento (entrevista) possa ter contribuído para aumentar as chances de os participantes terem respondido ao inventário conforme aquilo que consideravam socialmente aceito (Rios, 2010). Além disso, este posicionamento pode ser afetado por diferenças culturais, sendo que o conteúdo dos itens que medem a desejabilidade social pode não corresponder ao que é considerado como tal no contexto sociocultural brasileiro.

\section{Considerações Finais}

Este estudo trouxe importantes contribuições para a área da violência contra crianças, pois identificou fatores que parecem efetivamente correlacionados aos abusos físicos, considerados proximais em relação à emergência do fenômeno, especificamente aqueles ligados às características psicológicas do cuidador e aos problemas vividos nas interações.

Ademais, os resultados permitiram descrever as possíveis inter-relações entre as variáveis estudadas, com base no modelo do Processamento da Informação Social (Milner, 2000, 2003), denotando a importância dos processos básicos que parecem subjazer aos abusos, ainda que estudos futuros devam investigar quais variáveis contam mais para o potencial de risco e o modo como elas interagem. De toda forma, os resultados aqui obtidos dão pistas sobre as estratégias de intervenção a serem adotadas em casos de abuso físico, sendo que as técnicas da abordagem cognitivo-comportamental (ensino de habilidades de manejo do estresse e da raiva, de resolução de conflitos; reestruturação cognitiva) poderiam constituirse em ferramentas valiosas no tratamento dos cuidadores (Mammen et al., 2002; Runyon, Deblinger, Ryan, \& Thakkar-kolar, 2004).

Há, todavia, que se considerar que a obtenção dos dados no presente estudo apresentou alguns limites. $\mathrm{O}$ formato de entrevista adotado para a coleta, embora tenha contribuído no sentido de homogeneizar a qualidade da informação, pode ter tido um impacto significativo nos participantes aumentando o fornecimento de respostas baseadas no aspecto de desejabilidade social. Para diminuir esse efeito, seria necessário o estabelecimento de uma relação de maior confiança com os respondentes, o que seria possível somente no decorrer de interações mantidas em mais longo prazo. Ainda, tem-se o limite de o CAP não se encontrar totalmente adaptado à nossa cultura, não se dispondo, portanto, de normas que permitam aferir o quanto os escores obtidos constituem-se ou não em efetivo indicador de risco para o abuso físico. Por fim, o estudo realizado incidiu sobre um pequeno grupo de cuidadores notificados por abusos físicos, sendo necessário nas próximas investigações avaliar amostras mais numerosas e compostas por níveis de gravidade diferentes quanto às situações de abuso, uma vez que isso poderá indicar diferenças em processo básicos como a percepção, a avaliação e a interpretação, dependentemente da seriedade das situações concretas.

\section{Referências}

Associação Brasileira de Empresas de Pesquisa. (2003). Critério de Classificação Econômica Brasil. Retrieved January, 2006, from www.abep.org

Ateah, C. A., \& Durrant, J. E. (2005). Maternal use of physical punishment in response to child misbehavior: Implications for child abuse prevention. Child Abuse \& Neglect, 29, 169-185

Balge, K. A., \& Milner, J. S. (2000). Emotion recognition ability in mothers at high and low risk for child physical abuse. Child Abuse \& Neglect, 10(24), 1289-1298.

Belsky, J. (1993). Etiology of child maltreatment: A developmental-ecological analysis. Psychological Bulletin, 114, 413-434

Bérgamo, L. P. D., Pasian, S. R., Ávila de Mello, I. L. M., \& Bazon, M. R. (2009). O Inventário de Maus-Tratos Infantil: Estudo de Precisão e Validade. Avaliação Psicológica, 8(3), 425-435.

Black, D. A., Heyman, R. E., \& Slep, A. M. S. (2001). Risk factors for child physical abuse. Aggression and Violent Behavior, 6, 121-188.

Bringiotti, M. I. (1999). Maltrato infantil. Factores de riesgo para el maltrato físico en la poblacion infantil que concurre a las escuelas dependientes del Gobierno de la Ciudad de Buenos Aires. Madrid, España: Niño y Dávila.

Brown, J., Cohen, P., Johnson, J. G., \& Salzinger, S. (1998). A longitudinal analysis of risk factors for child maltreatment: Findings of a 17-years prospective study of officially recorded and self-reported child abuse and neglect. Child Abuse \& Neglect, 11(22), 1065-1078.

Coohey, C. (1996). Child maltreatment: Testing the social isolation hypothesis. Child Abuse \& Neglect, 20(3), 241-254.

De Antoni, C., Barone, L. R., \& Koller, S. H. (2007). Indicadores de risco e proteção em famílias fisicamente abusivas. Psicologia: Teoria e Pesquisa, 23(2), 125-132.

De Paúl, J., Asla, N., Pérez-Albéniz, A., \& Torres-Gómez, B. (2006). Impact of stress and mitigating information on evaluations, attributions, affect, disciplinary choices, and expectations of compliance in mothers at high and low risk for child physical abuse. Journal of Interpersonal Violence, 
21(8), 1018-1045.

De Paúl, J., Pérez-Albéniz, A., Guibert, M., Asla, N., \& Ormaechea, A. (2008). Dispositional empathy in neglectful mothers and mothers at high risk for child physical abuse. Journal of Interpersonal Violence, 23(5), 670-684.

DiLauro, M. D. (2004). Psychosocial factors associated with types of child maltreatment. Child Welfare, 53, 69-99.

Dopke, C. A., Lundahl, B.W., Dunsterville, E., \& Lovejoy, M. C. (2003). Interpretations of child compliance in individuals at high and low-risk for child physical abuse. Child Abuse \& Neglect, 27, 285-302.

Dopke, C. A., \& Milner, J. S. (2000). Impact of child noncompliance on stress appraisals, attributions, and disciplinary choices in mothers at high and low risk for child physical abuse. Child Abuse \& Neglect, 4(24), 493-504.

Francis, K. J., \& Wolfe, D. A. (2008). Cognitive and emotional differences between abusive and non-abusive fathers. Child Abuse \& Neglect, 32, 1127-1137.

Gomide, P. I. C. (2006). Inventário de Estilos Parentais - IEP. Modelo teórico- Manual de aplicação, apuração e interpretação. Rio de Janeiro, RJ: Vozes.

Haskett, M. E., Scott, S. S., Grant, R.,Ward, C. S., \& Robinson, C. (2003). Child-related cognitions and affective functioning of physically abusive and comparison parents. Child Abuse \& Neglect, 27, 663-686.

Heyman, R. E., \& Slep, A. M. S. (2000). Risk factors for family violence: Introduction to the special series. Aggression and Violent Behavior, 6, 115-119.

Hien, D., Cohen, L. R., Caldeira, N. A., Flom, P., \& Wasserman, G. (2010). Depression and anger as risk factors underlying the relationship between maternal substance and child abuse potential. Child Abuse \& Neglect, 34, 105-113.

Jackson, A. P. (2000). Maternal self-efficacy and children's influence on stress and parenting among single black mothers in poverty. Journal of Family Issues, 21(1), 3-16.

Mammen, O. K., Kolko, D. J., \& Pilkonis, P. A. (2002). Negative affect and parental aggression in child physical abuse. Child Abuse \& Neglect, 26, 407-424.

McElroy, E. M., \& Rodriguez, C. M. (2008). Mothers of children with externalizing behavior problems: Cognitive risk factors for abuse potential and discipline style and practices. Child Abuse \& Neglect, 32, 774-784.

Milner, J. S. (1986). The Child Abuse Potential Inventory: Manual ( $2^{\text {nd }}$ ed.). Webster, NC: Psytec Corporation.

Milner, J. S. (1990). An Interpretive Manual for the Child Abuse Potential Inventory. Webster, NC: Psytec Corporation.

Milner, J. S. (1994). Assessing physical child abuse risk: The Child Abuse Potential Inventory. Clinical Psychology Review, 6(14), 547-583.

Milner, J. S. (2000). Social information processing and child physical abuse: Theory and research. In D. J. Hansen \& C. Saptz (Eds.), Nebraska Symposium on Motivation: Vol. 46. Motivation and child maltreatment (pp. 39-84). Lincoln, NE: University of Nebraska Press.

Milner, J. S. (2003). Social information processing in high-risk and physically abusive parents. Child Abuse \& Neglect, 27, 7-20.

Milner, J. S., \& Chilamkurti, C. (1991). Physical child abuse: Perpetrator characteristics. Journal of Interpersonal Violence, 3(6), 345-366.

Montes, M. P., De Paúl, J., \& Milner, J. S. (2001). Evaluations, attributions, affect, and disciplinary choises in mothers at high and low risk for child physical abuse. Child Abuse \& Neglect,
25, 1015-1036.

Najmi, S., Bureau, J. F., Chen, D., \& Lyons-Ruth, K. (2009). Maternal attitudinal inflexibility: Longitudinal relations with mother-infant disrupted interaction and childhood hostileaggressive behavior problems. Child Abuse \& Neglect, 33, 924-932.

Ochotorena, J. P., \& Madariaga, M. I. A. (1996). Manual de protección infantil. Barcelona, España: Masson.

Palusci, V. J., Smith, E. G., \& Paneth, N. (2005). Predicting and responding to physical abuse in young children using NCANDS. Children and Youth Services Review, 27, 667-682.

Pittman, J. F., \& Buckley, R. R. (2006). Comparing maltreating fathers and mothers in terms of personal distress, interpersonal functioning, and perceptions of family climate. Child Abuse \& Neglect, 30, 481-496.

Ribas, A. F. P., Ribas, R. C., Jr., \& Valente, A. A. (2006). Bemestar emocional de mães e pais e o exercício do papel parental: Uma investigação empírica. Revista Brasileira Crescimento e Desenvolvimento Humano, 16(3), 28-38.

Rios, K. S. A. (2010). Inventário de Potencial de Abuso Infantil-CAP: Adaptação transcultural, fidedignidade e validade para o brasil. (Tese de Doutorado não publicada). Universidade Federal de São Carlos, SP.

Rodriguez, C. M. (2010). Personal contextual characteristics and cognitions. Predicting child abuse and disciplinary style. Journal of Interpersonal Violence, 25(2), 315-355.

Rodriguez, C. M., \& Richardson, M., J. (2007). Stress and Anger as contextual factors and preexisting cognitive schemas: Predicting parental child maltreatment risk. Child Maltreatment, 12(4), 325-337.

Runyon, M. K., Deblinger, E., Ryan, E. E., \& Thakkar-Kolar, R. (2004). An overview of child physical abuse: Developing an integrated parent-child cognitive-behavioral treatment approach. Trauma, Violence, \& Abuse, 5(1), 65-85.

Shay, N. L., \& Knutson, J. F. (2008). Maternal depression and trait anger as risk factors for escalated physical discipline. Child Maltreatment, 13(1), 39-49.

Suzuki, S. (2010). The effects of marital support, social network support, and parenting stress on parenting: Self-efficacy among mothers of young children in Japan. Journal of Early Childhood Research, 8(1), 40-66.

Vitolo, Y. L. C., Fleitlich-Bilyk, B., Goodman, R., \& Bordin, I. A. S. (2005). Crenças e atitudes educativas dos pais e problemas de saúde mental em escolares. Revista de Saúde Pública, 39(5), 716-724.

Woodward, L. J., \& Fergusson, D. M. (2002). Parent, child, and contextual predictors of childhood physical punishment. Infant and Child Development, 11, 213-235.

World Health Organization, \& International Society for Prevention of Child Abuse and Neglect. (2006). Preventing Child Maltreatment: A guide to taking action and generating evidence. Geneva, Switzerland: World Health Organization Press. 